Strokes are a severe complication of sickle cell anaemia in children and results in significant morbidity. The mainstay treatment for stroke prevention is chronic blood transfusions, however this is not without its risks and complications. By measuring transcranial Doppler velocity, at-risk patients can be identified and preventative treatment initiated. Hydroxycarbamide has been shown to reduce transcranial doppler velocities in sickle cell patients and therefore Hydroxycarbamide has been considered a potential alternative candidate to blood transfusions with less adverse effects.

Aim To assess whether hydroxycarbamide was effective in reducing transcranial doppler velocities and if this translated into efficient stroke prevention in children with sickle cell anaemia.

Search criteria The Cochrane database, PUBMED and Google scholar were searched. Prospective, Randomised Control and Observational trials were included.

Results Searches identified 6 eligible studies $(n=365)$. All studies unanimously showed an initial reduction in TCD velocities. One trial was able to show non-inferiority $(\mathrm{p}=8.82 * 10 \mathrm{E}-16)$ of hydroxycarbamide to chronic blood transfusions. Nevertheless, another study identified a reversion to abnormal TCD velocities after a mean follow up period of 1.1 years.

Conclusions In conclusion, Hydroxyurea appears to be useful in reducing TCD velocities in both abnormal (defined as TCD velocity $>200 \mathrm{~cm} / \mathrm{s})$ and conditional $(>180 \mathrm{~cm} / \mathrm{s})$ TCD velocities. In terms of stroke prevention, there is no significant decrease in occurrence or at least non-superiority. There is no consensus on the role of hydroxycarbamide as a prophylaxis of stroke.

\section{G367(P) COAGULATION SCREENS: INDICATIONS, ABNORMALITIES, MANAGEMENT AND FOLLOW UP IN A GENERAL PAEDAITRIC UNIT}

L McLaren, L Clarke, H Wayman, A McKie. Paediatric and Child Health Department, Royal Alexandra Hospital, NHS Greater Glasgow and Clyde, Paisley, UK

10.1136/archdischild-2018-rcpch.357

Aims To identify:

- Clinical indications for performing coagulation screens.

- Abnormalities found in coagulation screens.

- Management and follow up of abnormal coagulation screens.

Method A retrospective case note review of patients aged 016 years who had a coagulation screen during a 6 month period from 1 st January until 30th June 2017.

Results 219 samples were analysed. 45\% $(n=99)$ were male and $55 \%(n=120)$ female, aged 4 days to 16 years. The most common indication for performing a coagulation screen was presence of a non-blanching rash 32\% $(n=71)$. Bloody diarrhoea $7 \%(n=19)$, paracetamol overdose $7 \%(\mathrm{n}=15)$ and child protection medicals $5 \%(n=12)$ were the next most common indications.

$66.7 \%(n=146)$ of samples had at least one abnormality. 29\% $(n=64)$ of prothrombin times (PT), 25\% $(n=55)$ of activated partial thromboplastin time (APTT) and 35\% $(n=81)$ thrombin times (TT) were outwith the normal values for age. $62 \%$ $(n=91)$ had mildly abnormal results. Abnormal results were commonest in the 1 to 5 year old group $32 \%(n=47)$.

In those with mildly abnormal results repeat samples were arranged in $16 \%(n=15)$, whereas in coagulation screens with greater abnormalities repeat sampling was arranged in $55 \%$ $(n=30)$. Repeat samples were carried out at a median of 7 days from the original sample (range 0-129 days).
One patient received i.v. vitamin $\mathrm{K}$ for prolonged PT and 3 patients were referred to haematology. Two of who had family members with a clotting disorder and one had a new diagnosis of haemophilia B.

Conclusions

- The commonest reason for performing a coagulation screen was a non-blanching rash.

- The commonest abnormality is a prolonged TT, followed by PT then APTT.

- There is wide variability in follow up and timing of follow up of abnormal coagulation screens.

- Children with mildly abnormal screens are least likely to have repeat sampling.

Recommendation 1 . To develop a guideline to unify the follow up of abnormal coagulation screens.

\section{G368(P) CHILD WITH G6PD DEFICIENCY PRESENTED AS CONVULSION DUE TO ACQUIRED METHEMOGLOBINEMIA: CASE PRESENTATION}

S Ismail, P Gowda, A Yehia. Paediatrics, Oman Medical College, Muscat, Oman

\subsection{6/archdischild-2018-rcpch.358}

Introduction G6PD is an important enzyme in red blood cells and its deficiency renders erythrocytes susceptible to haemolysis under conditions of oxidative stress induced by oxidant drugs, ingestion of fava beans or its constituents, exposure to naphthalene, henna application, diabetic ketoacidosis and infection. It is an X-linked recessive condition, but homozygous females may also be affected. It is a common problem in some countries such the Arabian Peninsula, Turkey and the Middle-East; particularly in the Sultanate of Oman it affects $20 \%$ of male population.

The main presentation is acute intravascular hemolytic anaemia and neonatal jaundice. Other nonspecific features are of chronic anaemia when there is low-grade hemolysis.

Aim Raise awareness in the recognition of uncommon precipitants of hemolysis and explore further updated discussion on the efficient management of acute intravascular haemolysis due to G6PD deficiency and its sequel as well as prevention.

Case report An 8-year-old child presented with generalised tonic clonic convulsion lasting about $20 \mathrm{~min}$. On examination, he was pale and cyanosed with mild dehydration. Cardiac examination revealed a functional murmur. Abdominal examination was normal. Oxygen saturation was $\left(\mathrm{SpO}_{2}\right)$ at admission $(78 \%)$ which did not improve significantly with the administration of $10 \mathrm{~L} / \mathrm{min}$ oxygen via non-rebreather mask. Urine colour was red-brown. Acute hemolytic anaemia was suspected with the association of acquired methemaglobinemia based on clinical presentation and failure of oxygen therapy to improve $\mathrm{SpO}_{2}$. Initial investigations showed $\mathrm{Hb} 4.2 \mathrm{~g} / \mathrm{dL}$, high LDH, reticulocytes 16\%, Serum ferritin $3840 \mathrm{ug} / \mathrm{L}$ and blood gases showed $\mathrm{PaO}_{2} 92 \mathrm{~mm} \mathrm{Hg}$ and methaemoglobin $17 \%$. Urine was strongly positive for haemoglobinuria.

Careful history revealed that the day before presentation, the child was exposed to naphthalene at home. Naphthalene, available as small balls of different colours, is very attractive to children to play with and even putting the mouth as sweet and this precipitated hemolysis in this case.

Outcome The child received oxygen, forced alkaline diuresis and ascorbic acid therapy, and made full recovery after 5 days hospitalisation. 